\section{Kompass Ophthalmologie}

\title{
Das digitale Tagungsjahr 2020
}

Bettina Hohberger

Koordination

«OphthalmoCampus»
Liebe Leserinnen und Leser,

das Jahr 2020 war ein ganz besonders Jahr - wer hätte gedacht, dass sich innerhalb so kurzer Zeit ein ganzer Globus verändern kann. Masken wurden zum Alltagsutensil. Der Lockdown war sicherlich für viele eine Herausforderung, ob privat oder beruflich. Auch die Medizin stand und steht noch vor einer Herausforderung. Neben der Versorgung der COVID-19-Patienten, steht die Prävention und der Schutz der Bevölkerung und des medizinischen Personals im Fokus der Pandemiebekämpfung.

Auch die medizinische Fortbildung musste sich den neuen Gegebenheiten anpassen - zeigte es sich gerade in den vergangenen Monaten, wie wichtig es ist, dass sich die Medizin stetig weiterentwickelt und diese neuen Forschungsinhalte einen kurzen Weg «from bench to bedside» finden. Die Kongresswelt fand schnell eine Antwort auf die neue Situation und stellte ihre Veranstaltung auf digitale Formate um. So fand erstmalig vom 9. bis 11. Oktober, im «von Graefe-Jahr», die DOG virtuell statt. Parallel hierzu tagte die Julius Hirschberg Gesellschaft online. Neben diesen nationalen Fortbildungen, fanden auch Online-Kongresse im Ausland statt. Erstmalig hatte man nun durch den virtuellen Charakter die Option, von «zu Hause» auch diese Veranstaltungen zu besuchen.

Wir möchten Ihnen in der aktuellen Ausgabe des OphthalmoCampus einen Einblick in die vergangenen Monate geben, in denen sowohl im In- wie auch im Ausland die ersten virtuellen ophthalmologischen Kongresse starteten. Viel Spaß beim Lesen!
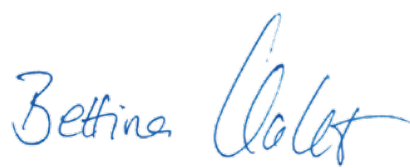

Ihre

Bettina Hohberger 


\section{Die DOG2020 online aus Sicht der AG Young DOG - ein besonderer Kongress}

Die Coronapandemie hat die gesamte Kongresslandschaft im Jahr 2020 auf den Kopf gestellt. Viele Kongresse mussten abgesagt, verschoben oder kurzfristig online angeboten werden. Auch die DOG2020 wurde kurzfristig in einen Onlinekongress umgewandelt. Damit war auch für die AG Young DOG die DOG2020 online eine sehr außergewöhnliche Veranstaltung, bei der zwar einige Initiativen Corona-bedingt ausfallen mussten, dafür aber neue ungeplante Formate hinzukamen. Im Nachhinein konnten trotz der neuen und großen Herausforderungen viele neue und spannende Impulse für zukünftige Kongresse gewonnen werden.

Aber erst einmal der Reihe nach: Für den DOG-Kongress in Berlin hatte die AG YoungDOG folgende Formate geplant: Das Doktorandensymposium 2020, auf dem die Doktorandenstipendiaten der DOG ihre Projekte vorstellen, ebenso das gemeinsame Frühstück mit den Stipendiaten. Ziel dieser beiden Veranstaltungen ist es, neben der Präsentation der Ergebnisse auch den Austausch zwischen den Doktoranden zu fördern und sich auch seitens der DOG mit den Doktoranden besser austauschen zu können. Diese beiden Maßnahmen mussten leider ausfallen.

Die komplexer werdenden Fragestellungen und Herausforderungen der Ophthalmologie erfordern eine stärkere Kooperation zwischen Grundlagenforschern und klinischen Wissenschaftlern. Diese Zusammenarbeit gilt es frühzeitig zu fördern. Als neue Maßnahme hatten wir daher letztes Jahr in Kooperation mit dem Young Researcher Vision Camp des European Vision Institute eine Sitzung etabliert, die sehr positiv angenommen wurde. Leider musste auch das Vision Camp Coronabedingt abgesagt werden und damit fiel auch das geplante Symposium aus. Wir hoffen aber, die Kooperation in Zukunft fortsetzen zu können. Das YoungDOG-Symposium mit dem Titel «Auf dem Weg ins Ausland - Optionen und erfolgreiche Planung» fand statt. Das übliche Get-together bei kleinen Snacks mit den Referenten, erfahrenen Wissenschaftlern, Mentoren sowie Chef- und Oberärzten musste selbstverständlich auch ausfallen. Sollte die DOG2021 wieder online durchgeführt werden müssen, hoffen wir mit der nun gewonnen Onlineerfahrung das Gettogether dann trotzdem durchführen zu können.

Darüber hinaus hat die AG Young DOG die Begrüßung und Einführung der Studierenden von Prof. Reinhard übernommen, der dies jahrelang sehr erfolgreich und hochmotiviert gemacht hatte. Das Online-Format ermöglichte eine andere Form der Begrüßung und des Austausches als die Frontalbegrüßung. Hier muss man sagen, dass beide Formate für die Begrüßung ihre Vor-und Nachteile haben. Weiterhin hatte Prof. Schiefer eine sehr spannende Führung

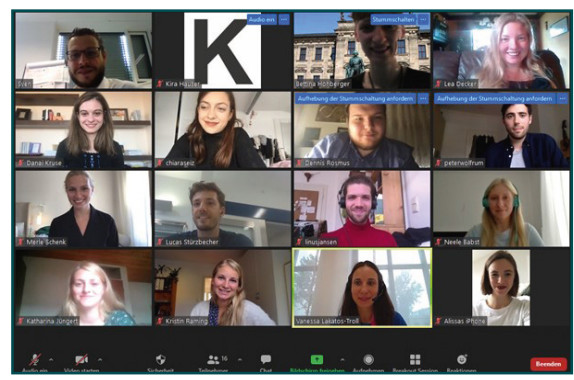

Screenshot des

Doktorandenstipendiatentreffens während der DOG2020 online. Foto: Sven Schnichels durch die Industrieausstellung für Studierende angeboten. Für diese möchten wir uns herzlich bei ihm bedanken. Ebenso gilt unser Dank dem Präsidenten und der Geschäftsstelle, die uns bei all unseren Aktivitäten online sehr unterstützt haben.

Als neues Format fand auch ein Onlinetreffen während der DOG mit den Doktorandenstipendiaten statt (siehe Foto). Hier zeigte sich, dass auch die Stipendiaten mit großen Herausforderungen während der Coronakrise konfrontiert sind. Dieses Onlinetreffen soll auf Wunsch der Studierenden zukünftig auch weiterhin stattfinden.

Um die digitale Interaktion innerhalb der AG YoungDOG während der fehlenden Präsenzveranstaltungen und darüber hinaus weiter zu fördern, verfügen wir nun über einen Instagramauftritt: @youngdog_official. Wir posten hier aktuelle und relevante Infos für den ophthalmologischen Nachwuchs.

Abschließend kann man sagen, dass die DOG2020 trotz der Coronakrise ein Erfolg war. Ein großer Dank an die hervorragende Organisation an dieser Stelle! Auch wenn leider liebgewonnene und erfolgreiche Formate nicht stattfinden konnten, wurden neue Formate eingeführt, die auch in Zeiten der Präsenzveranstaltungen weiterhin durchgeführt werden und die es wahrscheinlich sonst nicht gegeben hätte. Wir freuen uns daher auf ein spannendes 2021 und hoffen - obwohl die Online-Veranstaltungen alle sehr positiv waren - uns kommendes Jahr wieder in Berlin zu sehen!

Autor: Dr. Sven Schnichels, Universitäts-AugenklinikTübingen, Elfriede-Aulhorn-Str. 7, 72076 Tübingen, Deutschland, +49 707129 87888, sven. schnichels@med.uni-tuebingen.de

\section{Gastzugang für Studierende - «Schnuppermitgliedschaft»}

Es war ein lang gehegter Wunsch der AG Young DOG, dass auch Studierende in die DOG aufgenommen werden können. Dieses Thema wurde vor dem Hintergrund der Nachwuchsgewinnung und -sicherung immer drängender. Nach vielen zu klärenden rechtlichen Details (herzlichen Dank an die Geschäftsstelle und das GF-Präsidium an dieser Stelle) bietet die DOG nun eine sehr attraktive Möglichkeit für eine kostenlose 12-monatige «Schnuppermitgliedschaft» in der DOG an. Anschließend kann man zu vergünstigten Konditionen weiter 24 Monate den Gastzugang nutzen. Die Vollmitgliedschaft ist weiterhin erst mit erfolgreichem Abschluss eines entsprechenden Studiums möglich. Teilnehmen können Studierende ab dem 6. Semester. Weiterhin beinhaltet der Gastzugang die Fachzeitschrift «Der Ophthalmologe», freien Eintritt zum jährlichen DOG-Kongress, den Newsletters der AG Young DOG, die E-Mail-Informationen der Gesellschaft und die Möglichkeit zur Mitarbeit in der AG Young DOG. Wir hoffen, dass viele Studierende von der Mitgliedschaft gebraucht machen werden und wir diese so frühzeitig für die attraktive Karriere in der Augenheilkunde gewinnen können. Weitere Infos und Anmeldung sind auf der Homepage der DOG unter www.dog.org/?cat=296 zu finden. 


\section{Hat die virtuelle DOG dem Consilium Diagnosticum einen besonderen Charakter verliehen?}

Das Jahr 2020 ist für die ganze Welt aufgrund der Corona-Pandemie ein besonderes Jahr. Unser Leben wurde auf mehreren Ebenen beeinflusst und wird dies auch immer noch ... wer weiß, wie lange. Aufgrund dessen musste der DOG-Kongress dieses Jahr völlig anders gestaltet und für das Consilium Diagnosticum ein neues Format gefunden werden.

Das Jahr 2020 ist aber auch ein Jahr in dem jeder von uns ein Smartphone und ein Laptop zur Verfügung hat und Internet zum Alltag gehört. Die Voraussetzungen zur Durchführung eines virtuellen Kongresses waren also gegeben, aber die Umsetzung war neu und eine Herausforderung. Und trotz Prämiere hat das Konzept für das Consilium Diagnosticum hervorragend funktioniert, auch vor allem auf Grund der guten Vorbereitung.

Die Moderatoren, Herr Prof. Seitz und Herr Prof. Hoerhauf, die Techniker und wir Kollegen haben uns online getroffen und kennengelernt. In ganz Deutschland verteilt, von Erlangen bis Göttingen, von Berlin bis München saßen wir vor unseren Webcams und «sendeten» aus unseren Wohnzimmern zu Hause oder den Arztzimmern der Kliniken. Wir waren mehr als 20 Teilnehmer und die Koordination hätte chaotisch und problematisch werden können. Aber alles ist komplikationslos gelaufen, dank der klaren Vorgaben an uns und der guten Organisation. Jedem vorbereiteten und «abgespielten» Vortag folgte eine interessante Live-Diskussion mit Fragestellungen und Meinungsaustausch zwischen den Beteiligten, aber auch mit dem online Auditorium, das via Chat seine Kommentare und Fragen hinterlassen konnte.
Die Stimmung war gut und die Atmosphäre sehr angenehm, zum Teil vielleicht sogar auch entspannter als bei einer persönlichen Vorstellung in einem Hörsaal. Circa 600 Teilnehmer wurden für das Consilium Diagnosticum registriert und Fragen wurden bis zum Ende, fast 23:00 Uhr, gestellt. Kurz gesagt, darf man sagen, dass das Consilium Diagnosticum - und der gesamte DOG-Kongress 2020 - ein Erfolg gewesen sind! Dennoch, in einem «normalen» Jahr wären wir alle nach Berlin in unsere schöne Hauptstadt gereist, hätten uns schick angezogen, im Kongresszentrum getroffen und aufgeregt unsere Beiträge live gehalten. Danach hätten wir andere Vorträge gehört, an Kursen teilgenommen, in den Pausen die Industrieausstellung besucht, uns über Neuerungen informiert und nach den besten Werbegeschenken gesucht. Wir hätten den einen oder anderen wiedergetroffen und neue Kontakte oder Beziehungen wären entstanden. Beim EyeRun hätten wir uns unser T-Shirt verdient und Spaß gehabt. Der DOG Kongress «in echt» wäre sicher ein tolles Wochenende geworden.

Die Erfahrung des Consilium Diagnosticum 2020 war aber so gut, dass man sich dennoch daran gewöhnen könnte, einen Kongress online zu gestalten. Wie man sagt: aus der Not eine Tugend machen! Vielen Dank an Prof. Seitz und Prof. Hoerauf und alle, die zum Gelingen unseres Consilium Diagnosticum 2020 beigetragen haben! So schön es war, ich hoffe 2021 sehen wir uns alle wieder in Berlin!

Autorin: Maria Galimi, Augenklinik, Universitätsklinikum Erlangen, Schwabachanlage6,91054 Erlangen, Deutschland, maria.galimi@uk-erlangen.de

\section{Ophthalmologische Fortbildung aus Kiew online in die ganze Welt: Der dritte Ophthalmic Hub}

«Nichts ist so beständig wie der Wandel» - das wusste schon vor gut 2500 Jahren Heraklit von Ephesus (535-475 v. Chr.) und hat damit heute mehr denn je Recht:Die COVID-19-Pandemie hat unsere ganze Welt auf den Kopf gestellt, viele Planungen waren nur noch Schall und Rauch. Das galt auch für wissenschaftliche Kongresse: Sie wurden entweder rigoros abgesagt oder auf virtuelle Formate umgestellt. Ophthalmologische Gesellschaften, wie z.B.DOG und ESCRS, arrangierten sich mit der

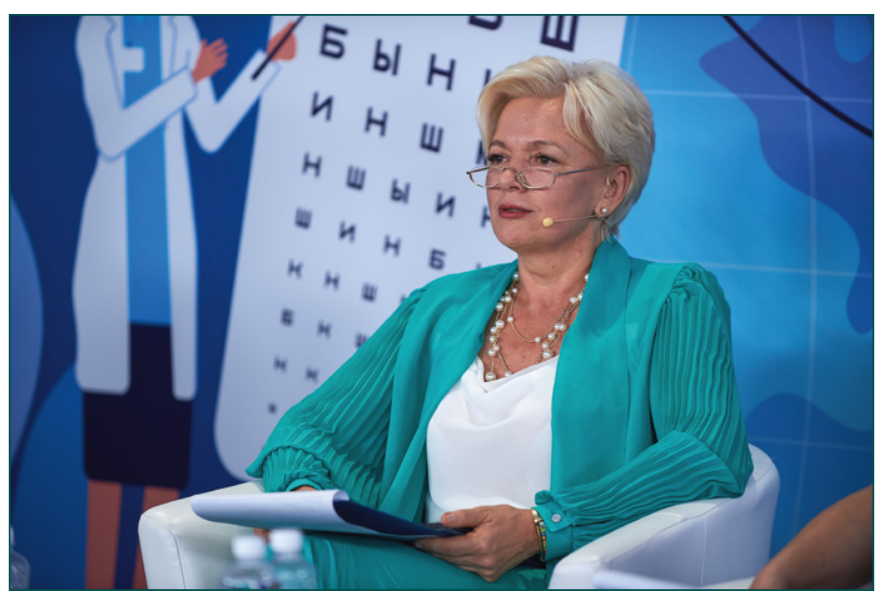

Prof. Oksana Vitovska, Initiatorin und Organisatorin des jährlichen Kongresses Ophthalmic Hub. Quelle: Dr. Sibylle Scholtz. neuen Situation und boten ihre großen Herbst-Kongresse virtuell an. Diesem Trend entsprechend fand auch der diesjährige, jährlich in Kiew (Ukraine) stattfindende internationale Ophthalmic Hub erstmals digital statt.

Im üblichen Rhythmus war der Ophthalmic Hub für März 2020 fixiert, dann wurde das Meeting auf den 30./31. Oktober verschoben. Frau Prof. Oksana Vitovska plante mittlerweile einen Hybridkongress, doch nur 2 Wochen vor dem Kongresstermin wurde auch diese Planung seitens der ukrainischen Gesetzgebung gekippt: nun durfte der Ophthalmic Hub ausschließlich als komplett virtuelle Veranstaltung stattfinden.

Nur das immense Engagement von Frau Vitovska und ihrem Team machte es möglich, allen diesen kurzfristigen Änderungen zu entsprechen und innerhalb weniger Tage einen professionellen und wissenschaftlich höchst attraktiven Online-Kongress zu realisieren. Diese Leistung wurde belohnt: Über 1500 nationale und internationale Teilnehmer waren bei diesem außergewöhnlichen Kongress virtuell in Kiew zu Gast und genossen auch dieses Jahr ophthalmologische Fortbildung auf internationalem Niveau. Über 100 Referenten bestritten in drei virtuellen Kongresshallen das wissenschaftliche Programm. 18 internationale Referenten aus 12 Ländern bestätigten die hohe wissenschaftliche Qualität auch des diesjährigen Kongresses.

Prof. Miguel Teus (Spanien) referierte über das chirurgische Vorgehen bei intraoperativer flacher Vorderkammer. Über «Bag in the 


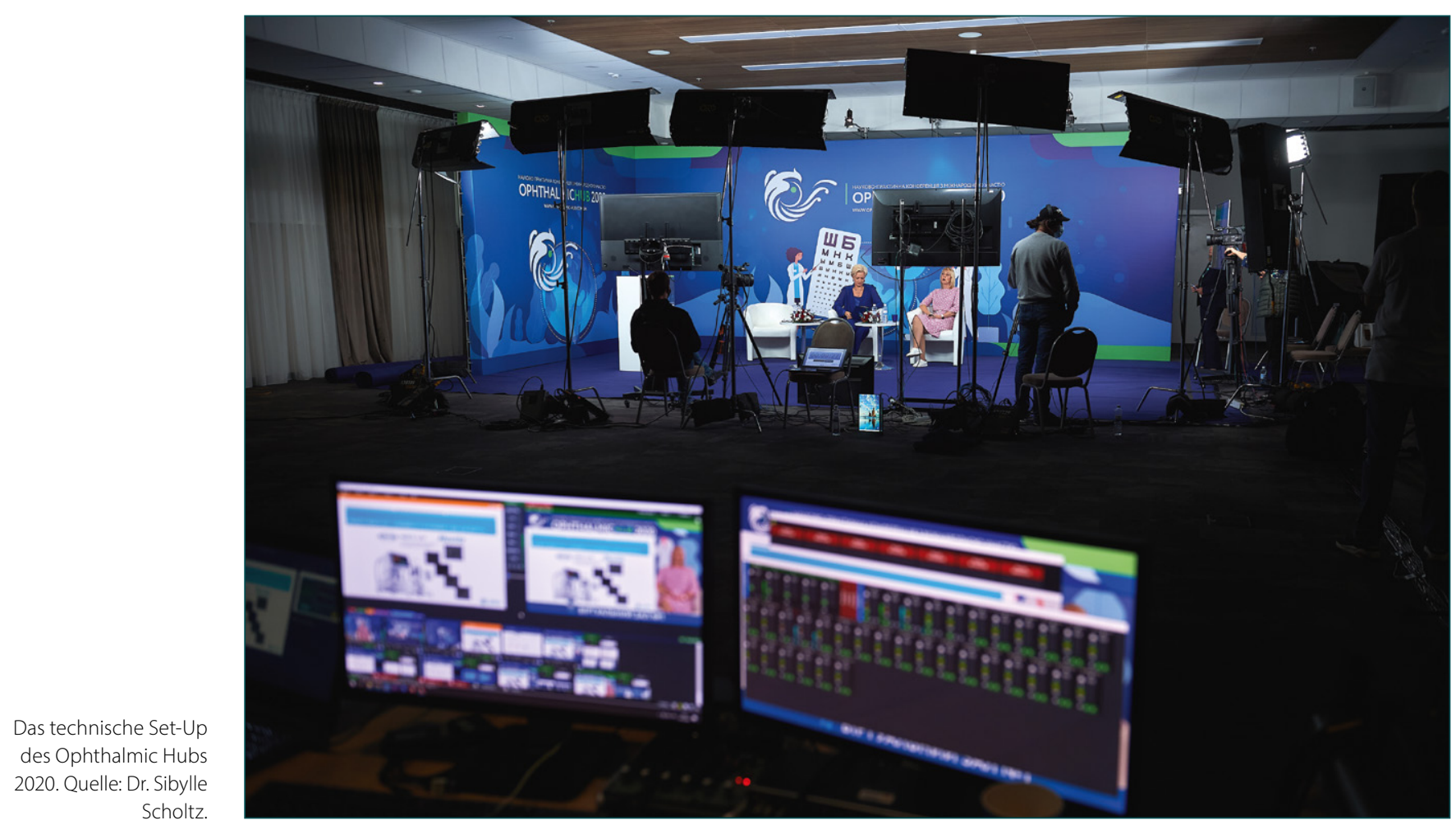

lens» in der Kataraktchirurgie sprach Prof. Marie-Jose Tassignon (Belgien). Aus Holland war Dr. Leonoor Koetsier mit ihrem Beitrag über die Therapie des Diabetes mellitus in den Niederlanden virtuell angereist. Gleich drei Referenten aus Portugal unterstützten den Ophthalmic Hub mit Berichten zum Einsatz von arzneimittelhaltigen Intraokularlinsen: Dr. Helena Filipe trug allgemein über die Thematik der kontrollierten Arzneimittelfreisetzung durch Intraokularlinsen vor. Dr. Ana Topete referierte über den Einsatz arzneimittelhaltiger IOL zur Prävention von Endophthalmitiden und anderen entzündlichen Reaktionen im Rahmen der Katarakt-Chirurgie. Nadia Toffoletto berichtete über arzneimittelbeschickte IOL zur Vorbeugung eines pseudophaken cystoiden Makula-Ödems.

Das Trockene Auge und praktische Empfehlungen zu einem modernen Therapieansatz war das Thema von Dr. Andrea Leonardi (Italien). Prof. Andrzej Grzybowski (Polen) berichtete über Kataraktchirurgie bei AMD-Patienten und die Versorgung durch Implantation von vergrößernden Sehhilfen. Wie man Therapieansätze für Glaukom-Patienten optimieren kann, war der Inhalt des Vortrages von Dr. Anastasios G. Konstas (Griechenland). Aus Deutschland sprachen Frank Krogmann zu «Alexander Ivanov (1836-1880), 1869 Prof. für Ophthalmologie in Kiew - Ein Europäischer Ophthalmopathologe par exellence» und Dr. Sibylle Scholtz über «Die Retina Risk App zur Einschätzung des individuellen Risikos der Progression einer Diabetischen Retinopathie».

Neben den Vorträgen und intensiven Diskussionen, die, wie auch in den Jahren zuvor, alle Bereiche der modernen Augenheilkunde abdeckten, wurde zudem praktisches Wissen in 15 Workshops vermittelt, z. B. mit der Masterclass zu «What can diplopia hide? Look at a simple question from the other side», die von Makar Ponomarev (Deutschland) als Coach betreut wurde, oder durch die Masterclass «Cataract surgery: Keep your hand on the pulse of innovation» unter der Obhut von Natalia Salcenko (Deutschland), Stephan Oestreich (Schweiz) und Delphine Lacambre (Frankreich).

Im Rahmen der kontinuierlichen professionellen Fortbildung für medizinische Ausbilder, die vom «International Council of Ophthalmology» unterstützt wird, wurde der Ophthalmic Hub dieses Jahr durch den Vortrag von Frau Prof. Helena Filipe (Lehrstuhl für Ophthalmologie, Universität Lissabon, Portugal) bereichert, mit dem sie für eine offene Kultur konstruktiver Rückmeldung warb und die dafür notwendigen Grundlagen erläuterte.

Professorin Vitovska setzte auch den diesjährigen Kongress als multidisziplinäres Format auf: Neun Spezialisten aus den Bereichen der Rheumatologie, Neurologie, Endokrinologie, Psychologie und dem Medizinrecht rundeten das ohnehin anspruchsvolle wissenschaftliche Fortbildungsprogramm ab. Die virtuelle Industrie-Ausstellung, die über 2000 Mal besucht wurde ergänzte das 2-tägige Treffen.

Frau Prof. Vitovskas großes Engagement und solide Vorbereitung haben sich mehr als bezahlt gemacht, ihr virtueller Kongress hat alle Erwartungen übertroffen: Innerhalb von 48 Stunden war die InternetSeite des Kongresses mehr als 25000-mal besucht worden. Auch die moderne Version des Ophthalmic Hub hat herausragende wissenschaftliche und didaktische Qualität bewiesen und sich als leistungsstärkste ophthalmologische Fortbildungsveranstaltung in der Ukraine etabliert. Wir sind gespannt, was uns der Ophthalmic Hub 2021 bieten wird - hoffentlich wird dieser dann wieder als Präsenzveranstaltung stattfinden können. Das wunderschöne Kiew und die gastfreundlichen Ukrainer sind immer eine Reise wert!

Mehr Informationen im Internet: ophthalmic-hub.com

Autor:Dr. Sibylle Scholtz, Ettlingen, Deutschland, sibylle.scholtz@gmx.de 


\section{«Nur wer sich ändert, bleibt sich treu» - der erste virtuelle Kongress der JULIUS-HIRSCHBERG-GESELLSCHAFT im Corona-Jahr 2020}

Wolff Biermanns Zitat traf mitten ins Herz der JULIUS-HIRSCHBERGGESELLSCHAFT (JHG), als mit dem Umschwenken der DOG auf einen Online-Kongress klarwurde, dass auch der zeitgleich stattfindende Kongress der JHG - von der Pandemie gezwungen - mitziehen muss. Nach dem mehrheitlichen Beschluss des Vorstandes der JHG stellten sich Frank Krogmann (Thüngersheim) und Dr. Sibylle Scholtz (Ettlingen) der völlig neuen Aufgabe, den Jahreskongress 2020 ebenfalls virtuell zu planen und durchzuführen. Dies gelang und das XXXIV. Zusammentreffen fand am 10. Oktober 2020 erstmalig online statt. Die JHG hatte zuvor beschlossen, das Zusammentreffen für alle Teilnehmer kostenlos und auch für Gäste offen anzubieten.

Das sonst üblicherweise vor dem Kongress stattfindende, erste Zusammentreffen in entspannter Atmosphäre wurde allgemein sehr vermisst. Doch dank des virtuellen Setups war es nicht nur Mitgliedern, denen eine lange Reise zu beschwerlich wäre, möglich, an der Tagung teilzunehmen, sondern auch internationalen Mitgliedern und Freunden der JHG. Auch dieses Jahr bot das Programm ophthalmo-historische Forschung auf höchstem Niveau.

Dieser erste virtuelle Kongress startete mit der Begrüßung durch die Organisatoren. Neben den Begrüßungsworten der aktuellen Obfrau Dr. Sibylle Scholtz und des Geschäftsführers Frank Krogmann wünschte auch überraschenderweise der DOG-Präsident Prof. Hans Hoerauf bei seinem spontanen Online-Besuch diesem besonderen Kongress ein gutes Gelingen.

\section{Die wissenschaftlichen Sitzungen}

Die erste wissenschaftliche Sitzung zur «Internationalen Ophthalmologie», unter dem Vorsitz von Prof. Dr. Oksana Vitovska (Kiew, Ukraine) und Dr. Frank Goes (Braschaat, Belgien) wurde mit der Keynote Lecture von Prof. Vitovska eröffnet. Sie sprach in «Ukrainian ophthalmology. From the past to the future» über die wichtigsten Errungenschaften in der Geschichte der ukrainischen Augenheilkunde. Die Referentin hat sich auf einige der bekanntesten Personen konzentriert, die nicht nur für die Entwicklung der nationalen, sondern auch der europäischen Augenheilkunde von großer Bedeutung waren. Neben dem Status der Augenheilkunde in der modernen Ukraine wurden die wichtigsten Möglichkeiten der Zusammenarbeit mit europäischen und anderen nationalen Gesellschaften sowie die künftige Entwicklung erörtert.

$\mathrm{Zu}$ «How Artists See the Eye» referierte Dr. Goes, denn die Augen eines Menschen verraten viel über die Gemütslage der jeweiligen Person. Das trifft auch zu, wenn Künstler Augen malen. Goes analysierte und diskutierte dies sehr anschaulich an Gemälden von Rubens, Rembrandt, Basquait, Manet, Lichtenstein, Van Eyck, Dali und anderen Künstlern.

Mit seinem Beitrag zur «Augenoperation in Tsingtau» schloss Prof. Dr. Guido Kluxen die dritte wissenschaftliche Sitzung ab. Er berichtete über Richard Wunsch, der am koreanischen Kaiserhof und in Tsingtau hauptsächlich Katarakte und Iridektomien operierte. Im Interview mit der DOG würdigte Prof. Dr. Hans Hoerauf die Verdienste des bedeutenden Augenarztes, Forschers und Lehrers

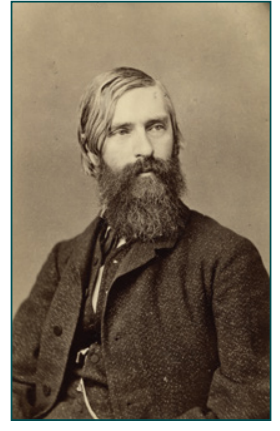

Im Jahr seines 150. Todestages beschäftigte sich auch die JHG auf ihrer Jahrestagung mit dem Begründer der modernen Augenheilkunde Albrecht von Graefe. Scan: H. Joop / Wikimedia Commons..

Albrecht von Graefe, der 1857 die DOG in Heidelberg gründete - die älteste augenärztliche Fachgesellschaft weltweit. «Wir sollten uns von Graefes Prinzipien ins Gedächtnis rufen, die empathisches ärztliches Wirken zum Wohle des Patienten und die Weitergabe seines Wissens und Könnens an seine vielen Schüler in den Vordergrund stellten», erklärte Hoerauf. Mit ihrer zweiten wissenschaftlichen Sitzung ehrte auch die JHG Albrecht von Graefe. Den Vorsitz dieser zentralen Sitzung hatten Prof. Guido Kluxen und Frank Krogmann.

Den Anfang machte Dr. Michael von Haugwitz (Esslingen, Deutschland) mit «Die Pathologie der Dioptrik und die Korrektion der Aberration. Allvar Gullstrand und Albrecht von Graefe». Der frühe Tod Albrecht von Graefes 1870 hat die Begegnung mit Allvar Gullstrand vereitelt. So konnte eine mögliche Etablierung der höheren Mathematik in der Forschung der Augenheilkunde durch die beiden Mathematiker und die «Ophthalmologische Gesellschaft» nicht gelingen. Der schwedische Augenarzt hatte im Rahmen seiner mathematischen Untersuchungen des Astigmatismus die pathologische Dioptrik entdeckt, die er im Laufe seines wissenschaftlichen Lebens zu einer Lehre der Dioptrik des menschlichen Auges erweiterte, wofür er 1911 mit dem Nobelpreis geehrt wurde. Der krönende klinische Abschluss seiner Untersuchung der pathologischen Dioptrik des Menschen ist die Erarbeitung der Theorie der Korrektion der Aberration der Strahlenbündel mit asphärischen, aberrationsaufhebenden brechenden Flächen von 1919.

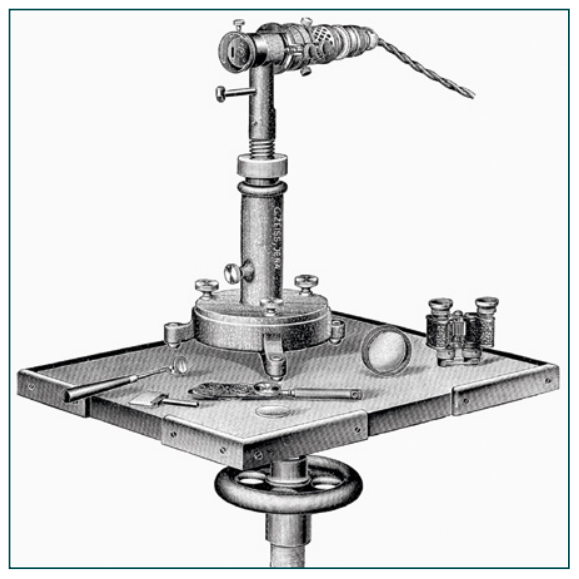

Neben seiner Lehre der Dioptrik des Auges zählt die Erfindung der ersten Nernst-Spaltlampe zu den wichtigsten Errungenschaften Gullstrands. Bearbeitung: MagentaGreen /Wikimedia Commons. 
Über «Dr. Friedrich Moritz Heymann (1828-1870) - Reformer der Augenheilkunde im Königreich Sachsen - Freund von Albrecht von Graefe» berichtete dieses Jahr PD Dr. Manfred Jähne (Schneeberg). Im Sommer 1850 lernte Heymann in Wien den gleichalten Albrecht von Graefe (1828-1870) kennen, es entwickelte sich eine lebenslange Freundschaft. Durch von Graefe fand Heymann seinen weiteren beruflichen Weg zum Augenarzt und hospitierte 1851 einige Monate bei von Graefe in Berlin. Heymann propagierte von Graefes Starschnitt und die Linearextraktion. Er starb 3 Monate nach von Graefe. Wenn Albrecht von Graefe als Reformator der Augenheilkunde allgemein gilt, so ist Friedrich Moritz Heymann der Reformer der Ophthalmologie in Dresden von 1851 bis 1870.

Über von Graefes Beteiligung am weltweiten ersten Augenärztekongress in Brüssel referierte Dr. Edward De Sutter (Kortrijk, Belgien) mit «Albrecht von Graefe und der «Congrès d'Ophtalmologie de Bruxelles 13, 14, 15 \& 16 septembre 1857»». Der erste internationale Kongress für Augenheilkunde fand 1857 in Brüssel statt. Albrecht von Graefe nahm teil, nachdem er eine Woche zuvor mit 12 Kollegen die erste deutsche Augenarztkonferenz in Heidelberg organisiert hatte. In diesem Zusammenhang sei auf die kürzlich erschienene von-Graefe-Biografie hingewiesen, die vom Medizinhistoriker Prof. Dr. Jens Martin Rohrbach veröffentlich wurde (Jens Martin Rohrbach: Zum 150. Todestag. Albrecht von Graefe (18281870). Das Gewissen der Augenheilkunde in Deutschland. 2020, Springer, Heidelberg).

Auch hatte das Team um Prof. Jutta Herde (Halle), Dr. Sibylle Scholtz, Prof. Achim Langenbucher (Homburg/Saar) und Dr. Udo Hennighausen (Hamburg) diverse ophthalmo-historische Publikationen über Albrecht von Graefe in internationalen Zeitschriften publiziert, mit denen ebenfalls das Andenken an Albrecht von Graefes 150. Todestag gewürdigt wurde, z. B. in der polnischen Zeitschrift «Przegląd Okulistyczny» (Polish Ophthalmology Review, S. 12, www.przegladokulistyczny.pl/uploads/att/43/3/1143/ PO_3-3020_small.pdf) und auch durch Prof. Oksana Vitovskas Initiative über die Ukrainische Ophthalmologen-Gesellschaft «Ukrainian Alliance of Ophthalmologists» (https://telegra.ph/Wreath-layingceremony-paying-tribute-to-the-150th-anniversary-of-the-death-ofAlbrecht-von-Graefe-A-role-model-in-medical-and-m-08-12).

Die dritte und letzte wissenschaftliche Sitzung «Varia» betreuten Dr. Frances Meier-Gibbons (Rapperswil, Schweiz) und Dr. Sibylle Scholtz als Vorsitzende, die mit dem Vortrag «Geschichte der Laserentwicklung in der Ophthalmologie: Von der Theorie zum täglichen Gebrauch» von Dr. Frances Meier-Gibbons eröffnet wurde. Zu Beginn des 20. Jahrhunderts entdeckten Max Planck und Albert Einstein die ersten Grundlagen für die Entwicklung eines Lasers. Planck postulierte, dass Energie nur in kleinen Teilchen, genannt Quanta, emittiert oder absorbiert werden kann. Einstein legte dann 1917 die eigentliche Grundlage zur Entwicklung der Laser mit der Theorie, dass Elektronen nicht nur spontan Licht emittieren und absorbieren können, sondern dazu stimuliert werden können, Licht einer spezifischen Wellenlänge zu emittieren. Ungefähr 50 Jahre später saß C. Townes in New York auf einer Parkbank und entwickelte die Idee eines Masers (micro- wave amplification by stimulated emission of radiation), der als Vorläufer des Lasers gilt. Die ersten Laserbehandlungen in der Ophthalmologie erfolgten 1960 mit einem Rubin-Laser, gefolgt von der Entwicklung eines Argon-Lasers für die Behandlung retinaler Erkrankungen, auch des Glaukoms. Die Entwicklung der Laser dauert bis heute an.

Den abschließenden Vortrag der dritten wissenschaftlichen Sitzung hielt Dr. Andreas Mettenleiter (Würzburg), er sprach über «Joseph Eduard (1807-1846) und Eduard Anton (1809-1877) - 2 Medizinerbrüder des Würzburger Ophthalmologen Robert von Welz (1814-1878)». Über den vielseitig medizinisch interessierten Ophthalmologen und Gründer der Würzburger Universitäts-Augenklinik Robert Ritter von Welz ist nur wenig bekannt. Dabei ist das be-

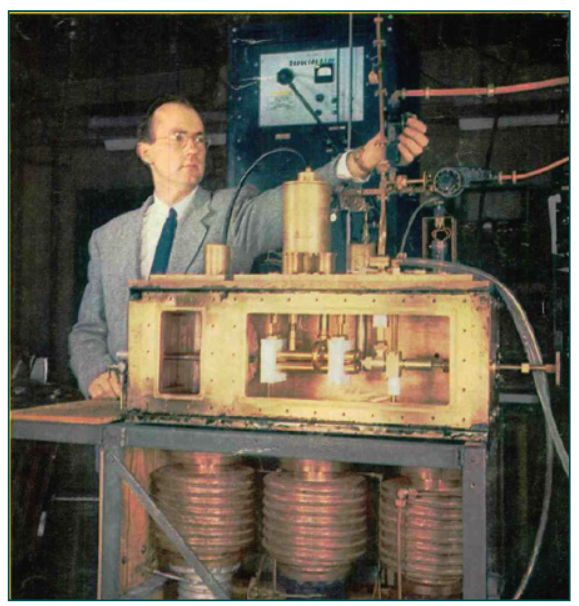

Charles Townes mit dem ersten Maser, den er 1953 zusammen mit James $P$. Gordon und H. J. Zeiger entwickelte.

Foto: Dan Rubin / Wikimedia Commons.

wegte Schicksal der beiden Brüder, die Medizin studierten und Ärzte wurden, sehr interessant und für das Verständnis der Persönlichkeit Robert von Welz' durchaus aufschlussreich.

\section{Ergebnisse der Arbeitsgruppe Ophthalmo-Ethik}

Trotz seiner vielfältigen Verpflichtungen seitens der DOG fand Prof. Dr. Gerd Geerling (Düsseldorf), Leiter der Arbeitsgruppe «Ophthalmo-Ethik in der JHG», Zeit aus dem unter seiner Initiative im letzten Jahr gegründeten Arbeitskreis der JHG zu berichten. Ethische Fragestellungen und Probleme sind oft eng mit historischen Entwicklungen verbunden. Sie können helfen, Ereignisse besser einzuordnen oder Orientierung für zukünftiges Handeln darzustellen. Um Fragen im Spannungsfeld von Ethik und Geschichte besser adressieren zu können, wurde der Arbeitskreis für Ethik in der Augenheilkunde auf der letzten Jahrestagung der JHG gegründet. Die ersten Schritte dieses neuen Arbeitskreises wurden von Geerling ausführlich dargestellt und diskutiert.

\section{Poster-Präsentation}

Auch dieses Jahr wurde die virtuelle Poster-Ausstellung von Dr. Frank Goes gründlich begutachtet und sehr fundiert kommentiert. In intensiver und enger Zusammenarbeit des zehnköpfigen Autorenteams, bestehend aus Dr. Sibylle Scholtz, Prof. Gerd Auffarth, Prof. Olaf Hellwinkel, Prof. Daniel Kampik, Prof. Philip Maier, Prof. 
Berthold Seitz, Thomas Wegner, Frank Krogmann, Katja Rosenbaum und Prof. Gerd Geerling, entstand eine grundlegende Arbeit, die sowohl die Geschichte des Hornhaut-Bankings allgemein als auch den großen Einsatz von Lions Clubs International hierbei beschreibt: «Die «Ritter im Kampf gegen die Blindheit〉 - Hornhautbanken und Lions Clubs International: Seit 1952 eine erfolgreiche Zusammenarbeit». Seit Eduard Zirm 1905 die erste erfolgreiche Keratoplastik durchführte, bestand die Notwendigkeit der Aufbewahrung von transplantierbaren Hornhäuten. Mit Helen Kellers Aufruf 1925, dass Lions die «Ritter im Kampf gegen die Blindheit» sein sollen, beteiligt sich Lions Clubs International weltweit an der Gründung von Lions Hornhautbanken, die erste wurde in New York (USA) im Jahr 1952 gegründet.

Der diesjährige Beitrag des Deutsch-Amerikanischen Teams (Dr. Sibylle Scholtz, Lee MacMorris und Prof. Achim Langenbucher) beschäftige sich mit «Eine Lösung, die ihr Problem sucht - 60 Jahre Laser-Technologie». Mit diesem Ausspruch kommentierte der amerikanische Physiker Theodore Harold Maiman (1927-2007) seine innerhalb kürzester Zeit die ganze Welt fundamental verändernde Erfindung: den Laser. Ohne auch nur annähernd zu ahnen, welch rasanten Erfolg und Einfluss sein kleines Gerät haben wird, das etwa $2 \mathrm{~kg}$ wog und bequem in eine Hand passte, stellte Maiman am 16. Mai 1960 der Welt den ersten Laser vor. Ohne diese revolutionäre Erfindung wären nicht nur Star Wars- und James Bond-Filme nicht das Gleiche, sondern auch die Medizin, und hier insbesondere die Augenheilkunde. Der Siegeszug des Lasers ist bis heute ungebrochen. Wenn man Obi-Wan Kenobis Segen auf heutige Patienten anpassen möchte, dann würde er wohl «Möge der Laser mit dir sein» lauten. Die Autoren berichteten über dieses Thema auch in «Ophthalmology Times Europe» (https://europe.ophthalmology times.com/view/60-years-of-laser-technology).

Das in Zusammenarbeit von weißrussischen und deutschen Wissenschaftlern bearbeitete Thema «Wissenschaft, Musik und Ophthalmologie, das aufregende Leben des Alexander Borodin ein Multitalent» wurde von Dr. Sibylle Scholtz, Prof. Tatsiana Imshanetskaya (Minsk, Belarus), Dr. Halina Sitnik (Minsk, Belarus), Barbara Geymayer (Burgkirchen, Deutschland) und Prof. Achim Langen-

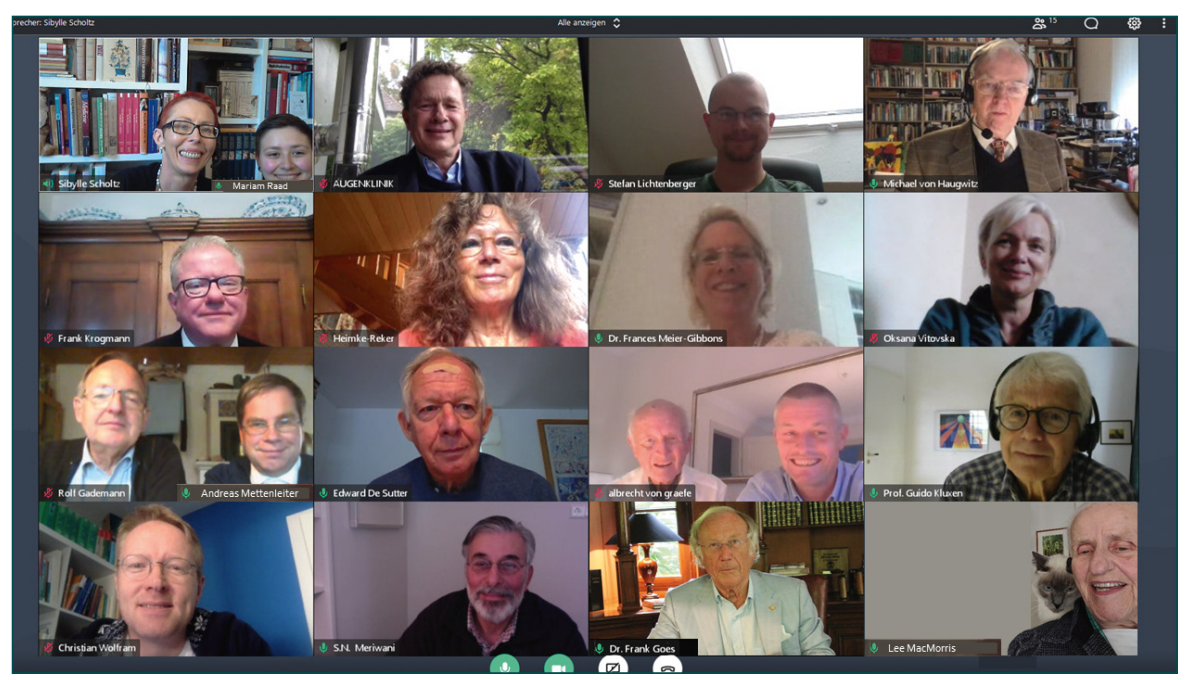

Das moderne Gruppenfoto vom virtuellen Kongress der JHG. Foto: Dr. Sibylle Scholtz. bucher präsentiert. Borodin war ein exzellenter Wissenschaftler und Arzt, weltberühmt wurde er für seine Musik. Alexander Porfiryevich Borodin (1833-1887) war ein bedeutender russischer Komponist des 19. Jahrhunderts. Seine berühmte Oper «Fürst Igor» gilt als eine der wichtigsten historischen, nationalen russischen Opern. Als Arzt nahm er 1857 am ersten internationalen Kongress für Ophthalmologen in Brüssel teil. Als einer von drei russischen Delegierten durfte er mit dem Chefophthalmologen der Militärmedizinischen Akademie St. Petersburg, Johann Kabath, zu dem Kongress reisen. Als Mitglied der berühmten «The Five» verwendete er russische folkloristische Themen, auch europäische Komponisten beeinflussten seine Arbeit. Borodins leidenschaftliche Musik und seine ungewöhnlichen Harmonien haben jüngere französische Komponisten, wie Debussy und Ravel, nachhaltig inspiriert. Mit «Vor einem halben Jahrhundert: Die Veröffentlichung der ersten Formel zur Berechnung der IOL-Stärke durch Gernet, Ostholt, Werner» erinnerten Dr. Sibylle Scholtz, Dr. Kristian Gerstmeyer, PD Dr. Timo Eppig, Frank Krogmann und Prof. Achim Langenbucher an die (fast) in Vergessenheit geratene IOL-Berechnungsformel. Harold Ridley implantierte 1949 die erste Intraokularlinse (IOL). In den folgenden 20 Jahren wurden sogenannte «Standard-IOLs» oder «Idem-IOLs» nach Faustregeln implantiert, ohne jegliche Ultraschallmessung des Auges. Erste Ideen zur präoperativen Berechnung der IOL-Stärke wurden 1967 von Svyatoslav Nikolayevich Fyodorov vorgestellt. Mit der «GOW-Formel» zur Berechnung der IOL-Brechkraft unter Nutzung von Ultraschall-Messergebnissen des Auges wurde eine weitaus zuverlässigere Möglichkeit zur individuellen Quantifizierung der IOL-Stärke vor der Kataraktoperation gefunden, die 1970 erstmalig von den Professoren Hermann Gernet, Heinrich Ostholt und Helmut Werner präsentiert und veröffentlicht wurde. Die GOW- bzw. Fyodorov-Formel wird als «Mutter aller Formeln» verstanden; sie ist die Grundlage für zahlreiche moderne, theoretisch-optische IOL-Berechnungsformeln, die heute verwendet werden.

Über «30 Jahre optische Kohärenztomographie des menschlichen Auges» berichteten Dr. Sibylle Scholtz, Frank Krogmann und Prof. Achim Langenbucher über die die erste OCT-Aufnahme einer menschlichen Retina in vivo. 1990 realisierte Adolf Fercher mithilfe der Weißlichtinterferometrie erstmals ein zweidimensionales Bild des Fundus eines lebenden menschlichen Auges. Fercher war der erste Forscher, der 1990 eine zweidimensionale in-vivo-Darstellung des Fundus eines menschlichen Auges entlang eines horizontalen Meridians auf der Grundlage von interferometrischen Tiefenscans mit weißem Licht präsentierte. Die optische Kohärenztomographie hat eine enorme wissenschaftliche und klinische Bedeutung. Sie bietet eine nicht-invasive Diagnostik, die in einer Vielzahl von medizinischen Geräten, insbesondere in der Augenheilkunde, verwendet wird. 
Die Details des Renaissance-Gemäldes «Der Blindensturz» (1568) von Pieter Bruegel dem Älteren erläutern Dr. Sibylle Scholtz, Paolo Pieracci, Frank Krogmann und Prof. Achim Langenbucher in ihrem Poster «Kunst, Bibel und Ophthalmologie: Pieter Bruegel's «Der Blindensturz»». Pieter Bruegel gilt als einer der bedeutendsten Künstler seiner Zeit. Das Gemälde zeigt eine Prozession von sechs Blinden, bei fünf davon ist jeweils eine andere Ursache für ihre Blindheit detailliert dargestellt. Bruegels Gemälde basiert auf dem biblischen Gleichnis vom Blinden, der Blinde führt (Matthäus 15:14 und in Lukas 6:39). Im Neuen Testament verglich Jesus häufig die, die körperlich blind waren, mit denen, die geistig

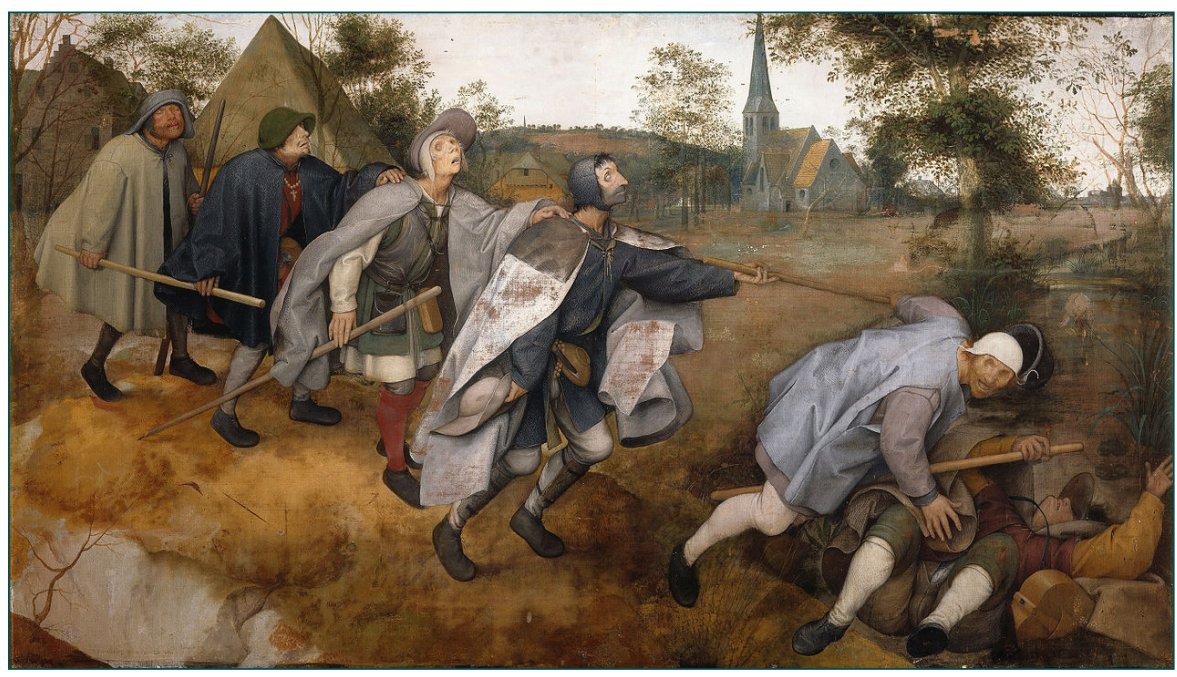

Pieter Bruegel der Ältere, «Der Blindensturz», 1568, Tempera auf Leinwand. Museo Nazionale di Capodimonte / Wikimedia Commons. blind waren. Schon vor Bruegel wurde dieses biblische Gleichnis verwendet, um andere Konfessionen anzuprangern. Luther nannte den Papst wiederholt «einen Führer für Blinde». Sein Gemälde bezieht sich weniger auf persönliches Fehlverhalten, als auf den Fall der christlichen Religion. «Der Blindensturz» kann aufgrund seiner Details und der Komposition als eines von Bruegels Meisterwerken angesehen werden. Es war auch Inspiration für andere Maler und Schriftsteller.

\section{Mitgliederversammlung}

Auch die diesjährige Mitgliederversammlung fand ungewohnt und dennoch erfolgreich online statt. Sie begann, wie üblich, mit der traurigen Aufgabe des Totengedenkens. Ganz besonders trauert die JHG um Univ.-Prof. Dr. med. univ. Hans Slezak, der am 25. Februar 2020 verstarb und das letzte lebende Gründungsmitglied der Gesellschaft war. Zwei Positionen des Vorstands mussten dieses Jahr gewählt werden. Wieder in den Vorstand wurde Prof. Jutta Herde und neu Dr. Andreas Mettenleiter gewählt. Eine Kassenprüfer-Position stand ebenfalls zur Wahl an, diese Funktion wurde mit Prof. Andreas Remky neu besetzt. Als Beisitzer wurde Univ.-Prof. Franz Daxecker berufen. Bei der sich der Generalversammlung anschließenden Vorstandssitzung wurde Dr. Sibylle Scholtz für das Jahr 2020/2021 erneut als Obfrau bestellt.
Der persönliche Kontakt, der direkte wissenschaftliche Austausch und das gemütliche, freundschaftliche Beisammensein sind unersetzlich. Andererseits: ein virtueller Kongress bietet mehr Teilnehmern die Möglichkeit dabei zu sein - 2020 hatte die JHG die vielleicht einmalige Möglichkeit, dies herauszufinden. Der Versuch gelang, die $J H G$ hat sich den aktuellen Bedingungen erfolgreich angepasst. Auch wenn virtuelle Kongresse sicher auch in Zukunft fester Bestandteil modernen Kongressgeschehens sein werden, freuen sich alle auf das nächste, das XXXV. Treffen der JHG, das vom 8.-10. Oktober 2021 unter der Ägide von Prof. Dr. Andreas Remky als Kongresspräsident in Regensburg stattfinden wird - hoffentlich analog!

Mehr Informationen zu den kommenden Kongressen und zur Gesellschaft sind hier zu erhalten:

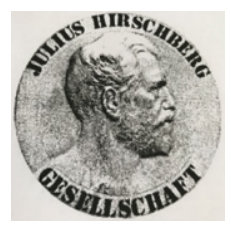

JULIUS-HIRSCHBERG-GESELLSCHAFT Frank Krogmann (Geschäftsführer) Kirchgasse 6, 97291 Thüngersheim, Deutschland, Tel.: 0049 9364/811543, Fax: 0049 9364/811559, Frank.Krogmann@t-online.de,www.jhg-online.org

Autoren: Dr. Sibylle Scholtz, Ettlingen, sibylle.scholtz@gmx.de; Mariam Raad, Karlsruhe, mariam.raad@gmx.de 